\title{
Analysis of fibre orientation effects on injection moulded components
}

\author{
F. O’Dowd, M. Lévesque*, M.D. Gilchrist" \\ Mechanical Engineering, University College Dublin, Ireland. \\ * Current address: Département de Génie Mécanique, École Polytechnique de Montréal, Canada.
}

\begin{abstract}
Fibre orientation, and thus the mechanical behaviour of short fibre reinforced thermoplastics (SFRTPs) depends greatly on the flow conditions in the mould. In order to design with SFRTPs, a thorough understanding of the processing conditions is required, together with the influence that the fibre orientation distribution (FOD) has upon the resulting mechanical properties. This work investigates the influence of primary injection moulding process parameters on the final properties of a simple planar injection-moulded component through DOE and ANOVA techniques. Four factors are seen to have greatest effect on the mechanical properties: cavity thickness, packing pressure, packing time and melt temperature. A systematic procedure is then employed to vary the levels of each factor, and the FOD and mechanical properties are studied. The final orientation is complex and is shown to vary throughout the part and depend heavily on the values selected for each parameter. Experimentally measured orientation results were then compared against those predicted by commercially available software for this planar geometry component. It was found that the predicted orientations were significantly over-predicted ( $34 \%$ on average), from a minimum of $15 \%$ for a $2 \mathrm{~mm}$ mould cavity up to $40 \%$ for the larger $5 \mathrm{~mm}$ cavity thickness. This discrepancy in turn leads to an over-prediction, of approximately $50 \%-60 \%$, between predicted and measured stiffness.
\end{abstract}

Keywords: Injection Moulding, Finite Element Simulation, Fibre Orientation

.Corresponding Author (michael.gilchrist@ucd.ie) 


\section{Introduction}

Recent years have seen a large rise in the number of applications for engineering thermoplastics within industry. Injection moulded parts are replacing structural metallic components since they are durable, offer good stiffness and strength to weight ratios, and can be produced for a lower total cost. The injection moulding process is almost fully automated, and its ability to quickly produce large volumes of net shape components is its main advantage. The route to manufacture, for an injection moulded component begins with establishing market/user requirements and a design brief, consisting of initial proposals and specifications. From this several solutions can be developed which satisfy the problem statement, before a detailed design phase can begin. Initially, the concept is given a physical presence, through the manufacture of a prototype. This embodiment stage provides a final check on function, tolerances and aesthetics and allows the performance of the concept to be evaluated. When satisfied with the product, the detailed design phase produces a complete description of the design suitable for manufacture. For injection moulding purposes, this involves material selection, mould design and choice of moulding parameters. This in turn leads to the manufacturing stages, where the mould is manufactured and the moulding machine set-up, through numerous iterations, to obtain a suitable product.

It has been shown, however, that up to $70 \%$ of production costs are determined during the concept formulation phase, even though the actual expense during this stage is very low [1]. In fact most of the cost is spent during the manufacturing phase, on items including materials, capital equipment, labour etc. Significant expense is also required for tooling and set-up of the manufacturing process, due to the fact that satisfactory component quality is difficult to achieve. Therefore, in order to address this situation, advantage must be taken of the initial stages to create both low cost products and process designs. It is vital that the component be designed with regard to performance and manufacturability before committing to expensive production costs. One such method used to evaluate the design process involves the use of computer simulations that predict the manufactured characteristics of components by injection moulding. Through the use of simulations, moulds can be accurately designed with balanced runners and adequate cooling channels.

The performance of an injection-moulded part is the result of a complex interaction of the inherent material properties and selected processing conditions. To increase stiffness and strength and reduce thermal expansion, short fibres are often incorporated in the resin. Glass fibre is by far the most widely used reinforcement, with 
practically all injection moulded thermoplastic resins available in glass-reinforced grades [2-4]. However, a key aspect of these fibre-reinforced materials is the complicated fibre orientation distribution produced during injection moulding. The mixture of glass fibres and molten polymer is injected under high pressure into a mould cavity, which fills in a matter of seconds. The resulting velocity fields and distributions of shear stresses that are generated during this mould filling process have been shown by a several studies, to have a profound influence on the fibre orientation structure, and thus the mechanical behaviour of such composites [5-10]. Typically a layered structure is found through-out the thickness of the moulding, and the orientation of each is highly dependent on the fibre characteristics, the melt flow pattern within the mould and the conditions used in the moulding process [11]. The number and depth of each layer has been the topic of a number of studies. In general, most agree that five layers exist forming a skin-shell-core structure [11,12]. However, some researchers have discovered the formation of up to nine layers from surface to surface [13].

As a consequence of the thermo-mechanical history imposed on the melt, the relative properties and thickness of each layer are dependent on the moulding conditions used and these determine the response under mechanical loading [14]. In order to design with SFRTPs, a thorough understanding of the processing conditions is required, together with the influence that the fibre orientation distribution (FOD) has upon the resulting mechanical properties. Given this complexity involved in filling of the mould and the intricacy with which the orientation distribution is formed, numerical prediction of the fibre orientation state becomes increasingly difficult. However, as the fibre orientation state is the foundation for the majority of calculation models, it is of great importance that it can be predicted accurately and efficiently. This paper reports the research carried out to investigate where and how current models for predicting and measuring fibre orientation lose accuracy. This research begins by optimising the injection moulding process for a particular component, through detailed factorial design of several operating parameters. Once this moulding process is optimised, through thickness views of the resulting fibre orientation are obtained using image analysis techniques. These orientations are then correlated with numerical predictions. Finally, the measured mechanical stiffnesses are compared against those predicted by simulation.

\section{Experimental}

Given the large number of processing parameters that define the injection moulding procedure, the most practical method of investigation is based on a design of experiments (DOE) technique. A full factorial design (represented 
by $2^{\mathrm{k}}$ ) is the preferred choice when all main and interaction effects are to be evaluated independently. However, even though it is possible to calculate all these effects, it does not imply that all are of appreciable size; also the number of runs increases geometrically as $\mathrm{k}$ is increased. Under such circumstances, fractional factorial designs provide a reasonable alternative that still allows the experimenter to get a clearer indication of major trends with relatively few runs, i.e. they exploit the inherent redundancy of the full factorial design. Choosing the design parameters and their levels is not a trivial task and even with a design tool such as factorial design, choosing the experimental parameters knowledgeably is crucial. Using part geometry, selected material and injection location, a moulding window analysis was used to calculate the preliminary process settings, as it quickly provides recommendations on injection time, mould temperature and melt temperature, which can then be used as input into the factorial design. For this investigation, it was decided to set each parameter at two levels, above and below the optimal settings, so as to explore as wide a region as possible, indicating major trends.

The eight most common and influential factors were selected from literature, each with two levels, thus requiring a design matrix with a minimum of 16 runs, Table 1 below. Therefore a $2_{\mathrm{IV}}^{8-4}$ design matrix was selected as the tool in which these factors would be investigated [15]. This type of matrix is ideal for examining the effects of main factors independently, since they are not associated with other interactions, as with other designs. The eight processing variables were examined for their influence on several quality parameters of the component, as characterised by mechanical performance and dimensional stability. Therefore, five properties were chosen and measured, namely, tensile strength, flexure strength, weight, impact strength and toughness, as quantified by the observed difference between peak energy absorbed and total energy during impact tests (i.e., $\mathrm{E}_{\mathrm{T}}-\mathrm{E}_{\mathrm{P}}$ ). These physical properties were all measured directly for five repetitions at each of the two levels for each of the eight factors indicated in Table 1. The measured results were analysed statistically as discussed below.

An Analysis of Variance (ANOVA) approach was used to evaluate the influence of each factor on the final outcome of product quality. This technique is basically an investigation into the equality of two or more population means, i.e., it determines what part of the total variation is due to error, to the factor itself or to interaction with other factors. From this work, it is evident that four factors, namely cavity thickness, packing pressure profile, packing time and melt temperature had the most effect on the quality of the specimen, as defined in terms of the measured responses of tensile, impact, and flexural strengths along with weight and toughness. To ensure accurate results, a 
check on the assumptions on which the fractional factorial model is based was performed by investigating the output from normal probability plots (not presented in the present paper) [16]. These are generated by plotting the cumulative frequency plot as a straight line: any deviations of the estimated contrasts from this line indicate that these effects cannot easily be explained by chance occurrence. The results from these probability plots confirm the results of the ANOVA analysis, i.e. that the four factors of cavity thickness, packing pressure profile, packing time and melt temperature were the most dominant. Table 2 below gives a summary of the statistical F-test results used in the ANOVA calculations.

A dumbbell shaped specimen, designed according to ISO 3167 and ISO 294-1, was used for all experiments. The dimensions of the part specify an overall length of $150 \mathrm{~mm}$, a gauge length of $80 \mathrm{~mm} \pm 2$, a gauge width of $10 \mathrm{~mm} \pm$ 0.2 , a tab width of $20 \mathrm{~mm} \pm 0.2$, and a radius of $20 \mathrm{~mm}$ between them. The material used throughout the duration of this project was a short glass fibre reinforced polypropylene manufactured by RTP Company. This material, RTP $105 \mathrm{PP}$, has a $30 \%$ E-glass fibre content with specific gravity of 1.11 . No information was supplied regarding the fibre length and measurements were obtained separately after resin burnout. Using a Mitutoyo Profile Projector (Model PJ300), the average fibre length was determined to be approximately 620 microns with radius of 12.5 microns. Polypropylene was selected as the base material, given the large quantity of components produced from this material.

A systematic procedure was then employed to manufacture a second set of samples, using these four primary factors, each at two levels. In this experiment the parameters are changed one at a time, with all others remaining at the optimum conditions. The order of the runs and the selected values of each parameter studied are given in Table 3 below. Similar to the method used in the preliminary factorial experiment, the runs are randomised as much as possible to prevent systematic bias creeping into the system. As each new run is initiated, the first $15-20$ samples were discarded allowing the system adequate time to reach operating conditions. Batches, prior to testing, were chosen at random from the specimens being produced for each run, again in an attempt to prevent errors being introduced into the results.

The fibre orientation of each of these samples was then measured, based on the observation that a section taken through the sample reveals an elliptical image of the fibre; and the shape of the fibre's cross section is strongly 
dependent on the degree of orientation. The orientation of a single fibre can be represented by a unit vector, $\mathbf{p}$, and its two orientation angles, $\theta$, the in-plane angle, and $\varphi$, the out-of-plane angle, Figure 1 . For a collection of fibres, the fibre orientation distribution function, $\psi(\theta, \varphi)$, is used to describe the probability of finding a fibre at a particular orientation. Although the orientation distribution function, $\psi$, is the most complete form, it is unwieldy and difficult to use, involving many calculations. An alternative method to describe the orientation state is given through the use of orientation tensors [17]. Orientation tensors are defined as the moments of the orientation distribution-function, and the second and fourth order orientations tensors can be described as follows:

$$
\begin{gathered}
\mathrm{a}_{\mathrm{ij}}=\int \mathrm{p}_{\mathrm{i}} \mathrm{p}_{\mathrm{j}} \psi(\vec{p}) \mathrm{d} \vec{p} \\
\mathrm{a}_{\mathrm{ijkl}}=\int \mathrm{p}_{\mathrm{i}} \mathrm{p}_{\mathrm{j}} \mathrm{p}_{\mathrm{k}} \mathrm{p}_{\mathrm{l}} \psi(\vec{p}) \mathrm{d} \vec{p}
\end{gathered}
$$

This second order tensor consists of nine components, however, due to symmetry $\left(a_{\mathrm{ij}}=\mathrm{a}_{\mathrm{ji}}\right)$ the number of components is reduced to six. Again using angle brackets to represent the average over all the fibres in a particular region, the tensor components can be written as:

$$
\begin{array}{ll}
\mathrm{a}_{11}^{\mathrm{k}}=<\sin ^{2} \theta^{\mathrm{k}} \cos ^{2} \phi^{\mathrm{k}}> & \mathrm{a}_{12}^{\mathrm{k}}=<\sin ^{2} \theta^{\mathrm{k}} \cos \phi^{\mathrm{k}} \sin \phi^{\mathrm{k}}> \\
\mathrm{a}_{13}^{\mathrm{k}}=<\sin \theta^{\mathrm{k}} \cos \theta^{\mathrm{k}} \cos \phi^{\mathrm{k}}> & \mathrm{a}_{22}^{\mathrm{k}}=<\sin ^{2} \theta^{\mathrm{k}} \sin ^{2} \phi^{\mathrm{k}}> \\
\mathrm{a}_{23}^{\mathrm{k}}=<\sin \theta^{\mathrm{k}} \cos \theta^{\mathrm{k}} \sin \phi^{\mathrm{k}}> & \mathrm{a}_{33}^{\mathrm{k}}=<\cos ^{2} \theta^{\mathrm{k}}>
\end{array}
$$

where $\mathrm{a}_{\mathrm{ij}}^{\mathrm{k}}$ denotes the orientation tensor for a single fibre. The main diagonal components indicate the magnitude of the fibre alignment in the direction of the reference frame, and the off-diagonal components show the rotation of the principal orientation axes with respect to the coordinate system. For a sample containing $\mathrm{N}$ fibres, the tensor components of the orientation distribution function are given as

$$
a_{i j}=\frac{\sum_{k=1}^{N} F_{k}\left(a_{i j}\right)_{k}}{\sum_{k=1}^{N} F_{k}},
$$


where the function $F_{k}$ is a weighting function for the $\mathrm{k}^{\text {th }}$ fibre. This is required as the probability of a finite fibre intersecting the cutting plane is dependent on the fibre length, $l$, the diameter, $\mathrm{d}$ and the angle that it makes with the plane, $\phi$. Fischer \& Eyerer [18] first introduced an orientation dependent correction factor based on the fibre concentration and aspect ratio. The correction factor in use today was developed by Konicek and Advani \& Tucker, summarised by Bay [5], and is based on the probability that a fibre would intersect the cross-section. To weight the measured data, it must be multiplied by the inverse of this probability, i.e.

$$
\mathrm{F}(\phi, l)=\frac{l}{l \cos \phi}, \quad \phi<\phi_{\mathrm{c}}
$$

The main limitation of measuring orientation from 2D polished surfaces is the ambiguity that exists in the in-plane angle $\phi$, i.e. the same elliptical image is produced by a fibre whose in-plane angle is $\left(180^{\circ}+\phi\right)$. However in many theoretical studies this is not a limitation of the technique [19], as only the signs of $\mathrm{a}_{23}$ and $\mathrm{a}_{13}$ cannot be determined, and absolute values are used instead. The elliptical parameters from each image, were then determined according to Stobie's second moments technique [20], which uses the object's second moments of inertia $\mathrm{M}_{\mathrm{xx}}, \mathrm{M}_{\mathrm{yy}}$ and $\mathrm{M}_{\mathrm{xy}}$ to calculate the elliptical parameters.

One final complication observed in obtaining the elliptical parameters from a particular section, is the difficulty in accurately measuring the in-plane angle, $\phi$ for near perpendicular cross sections [19, 21-23]. Clarke et al. [20] showed the emergence of an apparent frequency distribution in the in-plane angle, $\phi$ approximately between $15^{\circ}$ and $20^{\circ}$ when a section was taken perpendicular to the preferred fibre orientation, Figure 2. This apparent distribution arises from the difficulty in defining a circular object given the limited resolution of each image. However, once a section is taken, at an angle, the objects become well-defined ellipses, which removes the error associated with the pixellated nature of the image. In this work all the samples investigated have been taken at inclined sections, perpendicular to the preferred fibre direction and at an angle, $\alpha$ of $30^{\circ}$. The measured values can then be transformed back to the original section plane perpendicular to the preferred fibre orientation in order to determine the true values of $\theta$ and $\phi$. 
In this work all specimens were sectioned at three distinct locations namely, (i) $23 \mathrm{~mm}$ from the gate, (ii) $75 \mathrm{~mm}$ from the gate and (iii) $135 \mathrm{~mm}$ from the gate. Sections (i) and (iii) were chosen as the greatest change in orientation occurs at both the converging and diverging channels. This is appropriate, as the 2-dimensional flow in these channels will provide a challenging aspect for the numerical simulation. The middle section, section (ii), was selected as it accounts for the uniform gauge section of the specimen, and is practical when mechanical properties are investigated. Several images were required for each section, which were then combined to provide a full cross section of the sample, thus allowing the orientation distribution to be calculated. In the corresponding figures which are discussed later (Figures 6-14), sections (i), (ii) and (iii) are indicated in blue, red and black, respectively.

Figures 3 and 4 present in-situ SEM micrographs of a sample undergoing deformation as a result of an external load being applied to either end. The samples were also tested, using a Hounsfield 50K-S materials testing machine with a PS25/50 Extensometer, to measure the modulus of elasticity, in accordance with European standards EN ISO 5271 and EN ISO 527-2. The yield stress and proof stress were measured along with the elongation of the gauge length before failure, which allowed the initial tangent modulus to be determined and subsequently used to assess the accuracy of predicted values of moduli via Moldflow (see Table 4).

\section{Approaches to Modelling}

\subsection{Numerical Development}

This particular section considers the numerical scheme used to predict the fibre orientation and to calculate mechanical properties. As has already been shown, the properties of short fibre reinforced thermoplastics are closely related to the flow induced fibre orientation. Given the nature of the injection moulding process, it is extremely difficult to control the orientation during mould filling, which leads to a preferential orientation throughout the part. It is essential that the final properties of an injection-moulded component can be accurately and reliably predicted during the concept stage of developing a new component. Otherwise, a worst-case scenario must be assumed leading to over-engineering and corresponding high production costs.

Of the three regions that exist during injection moulding, i.e. the gate region, the lubrication/cavity region and the fountain region; the lubrication region is assumed to dominate the filling process. As no significant out-of-plane 
velocities exist in this region, it can be appropriately modelled using the Generalised Hele-Shaw formulations. The following assumptions are made regarding the filling of injection moulds:

- The main requirement is that $\mathrm{L}>>2 b$, where $\mathrm{L}$ is the length of the cavity in the flow direction and $2 \mathrm{~b}$ is the cavity thickness.

- No flow occurs in the thickness direction, thus the temperature, velocity and orientation fields are symmetric about the midplane $(\mathrm{z}=0)$.

- Inertia, surface tension and body forces are neglected, as well as the in-plane stress in the momentum equation.

- The flow is incompressible and inelastic.

The flow at the gate region is three-dimensional, and cannot be represented by Hele-Shaw type flow. For velocity and temperature profiles this region is normally neglected as it has little influence on these parameters [5]. Gupta \& Wang [24] also show that this region has only a minute effect on the fibre orientation (mostly $\mathrm{a}_{11}$ ) and this effect diminishes further from the gate. Papathanasiou [25] reviewed the work of a number of studies regarding the overall effect that the gate region imposed on the final orientation of injection-moulded parts, and concluded that predicted orientation distributions are insensitive to gate location or geometry. However, the above research was carried out on thin cavity components, and it must be noted that as the thickness of the part increases so too does the influence of the gate geometry on the final orientation, as in the present work. It has been suggested by Bay \& Tucker [6] that the effects of the gate region, if so required, be replaced by appropriate boundary conditions on entry to the lubrication region.

Correspondingly, the fountain flow region cannot be simulated by Hele-Shaw flow either, as the gapwise velocity is comparable to the in-plane velocity. Bay \& Tucker [7] discovered that the fountain flow model is responsible for the prediction of the skin layer in moulded parts. This layer, a dip in the orientation followed by a rise, as one moves towards the wall is predicted well, however, the orientation within this dip is not. They suggest the finite size of fibres, and non-Newtonian and non-isothermal effects are the cause for the inconsistency. Tucker [26] investigated the flow of fibre suspensions in narrow channels and concluded that the flow could be decoupled from the fibre orientation. Depending on a dimensionless particle number and the out-of-plane orientation, four separate flow regimes were discovered. One such flow permits the lubrication theory to be decoupled from orientation 
provided the channel is narrow enough such that the out-of-plane orientation does not contribute to the gapwise shear stresses. Although this approach is employed in the majority of injection-moulding simulations, it is not true, since the very presence of fibres results in a melt with an anisotropic viscosity, which changes as the fibre orientation changes. Advani \& Tucker [17] describe a suspension with 20\% volume fraction of fibres (each with an aspect ratio of 20) where the ratio of elongational viscosity to shear viscosity is 16 . For such a significant anisotropy, flow and orientation should be coupled both for the prediction of orientation and constitutive equations. Nevertheless, it is assumed that the flow can be decoupled from the fibre orientation, and is generally accepted in most theoretical work. The governing equations for flow are now identical to the Generalised Hele-Shaw flow formulations, and were solved by Hieber \& Shen [27].

Numerical predictions of fibre orientation during mould filling are based on Jeffery's equation of motion for a rigid ellipsoidal particle [28]. A rotary diffusion term, proposed by Folgar and Tucker [29], is added to this to account for fibre interactions, and is of the form:

$$
\frac{D a_{i j}}{D t}=-1 / 2\left(\omega_{i k} a_{k j}-a_{i k} \omega_{k j}\right)+1 / 2 \lambda\left(\dot{\gamma}_{i k} a_{k j}+a_{i k} \dot{\gamma}_{k j}-2 \dot{\gamma}_{k l} a_{i j k l}\right)+2 C_{I} \dot{\gamma}\left(\delta_{i j}-3 a_{i j}\right)
$$

where $C_{I}$ is a constant called the fiber interaction coefficient. Two main problems occur when trying to use this equation to predict fibre orientation. Firstly, the equation is not closed, i.e., the fourth order tensor, $\mathrm{a}_{\mathrm{ijk} k}$, is required to calculate the second order derivative. Secondly, the interaction coefficient must be determined for a particular material.

In the first instance, the software uses a closure approximation to approximate the fourth order tensor in terms of a second order tensor. Several approximations exist and have been tested in detail by Advani [30]. Advani \& Tucker's hybrid closure along with Cintra \& Tucker's family of orthotropic closure approximations can be selected according to flow regime and interaction coefficients, and the calculation is performed at up to 25 different layers, of each element, in order to capture the heterogeneous nature across the part. For the present work an orthotropic approximation is implemented [31]. To determine the fibre interaction coefficient, the work of Bay [5] was employed, who found for several materials a semi-empirical relationship of the form:

$$
\mathrm{C}_{\mathrm{I}}=\mathrm{A} \exp \left(\mathrm{B} \frac{\mathrm{cL}}{\mathrm{d}}\right)
$$


where $\mathrm{A}$ and $\mathrm{B}$ are constants, $\mathrm{c}$ is the volume fraction of fibres in the material, $\mathrm{L}$ is the average length of the fibres and $\mathrm{d}$ is the fiber diameter. In addition to this, the software also includes a revised model for the fibre-fibre interaction parameter. An extra term called the thickness moment of interaction, $\mathrm{D}_{z}$, is introduced to the model in an attempt to reduce the predicted out-of-plane orientation, caused by the closure approximation. Adjusting the magnitude of $\mathrm{D}_{z}$ controls the randomising effect in the out-of-plane orientation due to the fibre interaction. Thus by decreasing this $\mathrm{D}_{z}$ parameter, the predicted out-of-plane orientation is reduced and the core layer thickness is increased. It is also worth noting that setting $C_{I}=0.0$ returns the model to the original Jeffery form, and by setting $\mathrm{D}_{z}=1.0$ gives the Tucker-Folgar model for fibre interaction. Tests undertaken by Moldflow have shown that this revised model of orientation $\left(\mathrm{D}_{z}=0.01\right)$ along with Bay's relation for the interaction coefficient offers substantially improved orientation prediction over the other models.

Calculation of mechanical properties of short fibre composites begins with a micromechanics model for perfectly aligned fibres, then with solution of the fibre orientation of composites, can be taken to an orientation averaging procedure to determine the effective properties of the real composite. Since the properties of fibre-filled composites depend strongly on the flow fields during filling and packing, accurate modelling of these stages is required to obtain constructive solutions. Several micromechanics models have been developed, which are all based on the following assumptions:

- The fibre and the matrix remain elastic in their mechanical response, the matrix is isotropic and the fibres are either isotropic or transversely isotropic.

- The interface between the fibres and the matrix is perfect, that way interfacial slip, fibre/matrix debonding or matrix micro cracking is not considered.

- All fibres are identical in shape and size, and can be represented by an aspect ratio $l /$ d. Also, no axial force is transmitted through the fibre ends.

A number of these models can be implemented in the software, such as Cox, Krenchel and Halpin-Tsai equations, however, it has been found that the Tandon-Weng formulations give the best predictions of elastic properties for short fibre reinforced composites. Once the orientation tensor and the effective properties of perfectly aligned composites are known the final calculation is based on orientation averaging, as outlined by Advani \& Tucker [17]. 
This particular step attempts to adjust properties calculated for the fully aligned material to account for the anisotropic properties of injection moulded composites.

\subsection{Model Representation}

The model geometry was depicted using dedicated CAD software, and then transferred into the simulation package using an IGES file format. Two mesh types were available, a midplane mesh and a fusion mesh. The fusion mesh uses shell elements on the outside surfaces of a component's geometry to accomplish simulation. This surface mesh analysis works by simulating the flow of the melt on both the top and bottom parts of the mould cavity. However, it is limited by the fact that it cannot be used with stress-analysis packages. The midplane mesh consists of a web of 3-noded triangular elements and forms a 2D representation of a solid model, using the thickness information, during a moulding analysis.

Mesh density is the number of elements per unit area. In general, the more elements in the mesh, the more accurate the analysis results, at the expense of longer calculation times. The effects of various mesh densities on property calculations were investigated by $\mathrm{Ni}$ [32]; who showed that a large improvement in the accuracy of results can be realised by reducing the element size. Various mesh densities were examined for the specimen geometry of the present work, and the influence of this can be clearly seen during mould filling. Element edge lengths of $0.75,1,2$, 3 and $5 \mathrm{~mm}$ were investigated and the difference in pressure distribution at the end of filling is shown in Figure 5. The pressure increases during the filling stage until it reaches a maximum when the cavity is filled. The pressure then drops off as the part begins to solidify and shrink, and eventually decreases to zero. The pressure predicted using the $3 \mathrm{~mm}$ and $5 \mathrm{~mm}$ element meshes were on average $15 \mathrm{MPa}(13 \%)$ higher than the $1 \mathrm{~mm}$ mesh, while the $2 \mathrm{~mm}$ mesh had a better performance in that it was an average of $6 \mathrm{MPa}(5 \%)$ higher than the $1 \mathrm{~mm}$ element mesh, while no real improvement was obtained by further refinement, i.e. a $0.75 \mathrm{~mm}$ was marginally better providing a pressure of $1.5 \mathrm{MPa}(1.3 \%)$ lower than the $1 \mathrm{~mm}$ mesh density. These results are in agreement with the work of $\mathrm{Ni}$ [32] who showed that the pressure decreased as the element size was reduced, i.e. higher mesh density.

The mesh selected for all simulations consists of elements with an edge length of $2 \mathrm{~mm}$. This choice represents a compromise between a coarse mesh, providing rough simulations, and a very fine mesh with excessively long computational times. Several pre-processing operations were then required to edit and validate the mesh before 
analysis can begin. This was necessary to correct such issues as high aspect ratios, overlapping elements, mesh orientation, connectivity and non-manifold edges. Through successive iterations, the model was fine-tuned, until geometry, mesh density and gate and runner meshing were adequate to predict physical behaviour in short computation times. Next, material data and injection moulding machine specifications were specified for the software, so as to produce realistic and accurate predictions. Simulated results from filling and packing analyses were then correlated with actual short shots, as a means of validating the FE model.

The deflection of the specimen under loads was also investigated, by applying specific constraints and loads to the model. The resulting deflections and stresses were then calculated, allowing the tensile modulus to be obtained, which could then be correlated against the corresponding mechanical tests.

\section{4. $\quad$ Results \& Discussion}

Figures 6 and 7 below present the results of both the measured and predicted fibre distributions, for the $2 \mathrm{~mm}$ and $5 \mathrm{~mm}$ thick cavities, in the flow direction only, $\mathrm{a}_{11}$, i.e. along the length of the cavity. The data predicted from numerical simulations suggests a much greater alignment in the flow direction, for all sections, than actually measured. The form of the $2 \mathrm{~mm}$ cavity results is mostly in agreement with measured orientations, particularly for the mid-section and the section furthest from the gate, on average being approximately $15 \%$ over-predicted. Results for the $5 \mathrm{~mm}$ cavity, do not show as good agreement between predicted and measured orientation distributions. The second set of samples, Figures 8 and 9, give the results obtained for differing packing pressure profiles of $50 \%$ and $100 \%$. Similar to the results for the $5 \mathrm{~mm}$ cavity, a large disparity can be seen for the results obtained for the packing pressure profiles, although the distributions do improve for the larger pressure profile. As expected, given the results attained from the preliminary experimental work, the measured orientation increases for all sections taken. Added to this is the fact that the simulations do not consider an increase in packing pressure will affect the orientation distributions, as no real distinction is obvious between numerical calculations. From previous experiments undertaken it is apparent that packing pressure has a significant role in shaping the components ultimate properties. This influence is clearly not visible in predicted results.

As with the previous set of results, Figures 10 and 11 show that the numerical calculations again assume that packing time has little or no effect on the orientation distribution. Similar to the preceding results, packing time 
also has an influence on the orientation distribution. From Figure 10, a clear distinction between results is visible. Measured data exhibits the typical structure, observed in many of the samples thus far, albeit slightly shifted to the top edge of the part given the geometry of the edge gate. In general the final set of results illustrate quite a visible difference between predicted and measured data for both sets of results at $225^{\circ} \mathrm{C}$ and $265^{\circ} \mathrm{C}$ melt temperatures, Figures 12 and 13. For the aforementioned $225^{\circ} \mathrm{C}$ temperature, there is an average variation of $31 \%$ between numerical predictions and measured values, which increases to approximately $34 \%$ for the higher temperature setting. For the most part this is due to the increase in the out-of-plane orientation measured for the end section, furthest from the gate. In this section alone, the average orientation drops from $\mathrm{a}_{11}=0.65$ for the lower temperature, to $\mathrm{a}_{11}=0.45$ for $265^{\circ} \mathrm{C}$. This reduction in orientation is caused by a combination of the diverging channel preceding this section and the reduced melt viscosity given the greater temperature of the melt.

All measured results illustrate a 'typical' skin-shell-core structure, i.e. random orientation at the surface, due to instantaneous freezing, increasing as one moves through the thickness with increasing shear rates, and eventually dropping off in the centre, through a combination of a flatter velocity profile and plug flow, thus providing low levels of orientation. On the other hand, predicted results for the most part, as well as having greater alignment with the flow, do not contain this skin-shell-core structure, with the exception of the section near the end. Given the discrepancy between results, significant disagreements between mechanical property calculations can only be expected.

One of the most visible differences between the numerically predicted and experimentally measured orientation distributions was the asymmetric nature of the distribution for actual components. The type of gate used may explain this, as the edge gate is attached along the top length sample. Since the gate is not connected to one entire side of the sample, but only to one-third of the edge, it is not surprising that the orientation is altered to this side. Since the gate region could not be included in the numerical scheme, the predicted results were all symmetrical about the mid-plane. Together with the fact that the fountain flow region was not accounted for in the predicted results, significant disagreement between simulation and experiment was apparent in many instances. In some samples the skin layer is apparent, represented by a slight dip in the orientation followed by a rise, as one moves closer to the mould wall; this was never predicted by the simulations. This was also apparent in the fact that the best agreement between results was obtained nearest the mould wall, and in several instances, the simulated 
orientation parameter was under-predicted. Hence all simulations predicted the same reduced orientation, towards a more random orientation, where this skin layer was actually present in practice.

The Hele-Shaw formulations are another source of error, as it can be seen that for the narrow $2 \mathrm{~mm}$ cavity, a $15 \%$ disparity exists between numerical and measured results for orientation values. This increases to $40 \%$ when data is compared for the $5 \mathrm{~mm}$ cavity, thus suggesting that the limit of this theory has well and truly been passed. A linear increase in error is obtained as the cavity thickness increases, considering that for the $4 \mathrm{~mm}$ cavity an average error of $31 \%$ was obtained. Overall, it was found that the measured orientation was less than that predicted by approximately $34 \%$ for all cavities and conditions.

The values for the simulated and experimental calculations are presented in Table 4, along with the percent difference between these values. Given that the accuracy of elastic constant predictions is reliant on acquiring accurate fibre orientation distributions, it is no surprise that the mechanical property calculations were overpredicted by amounts varying from $52 \%$ to $59 \%$, especially considering the errors between measurements and simulations. These calculations are also dependent on assumptions and approximations used in mechanical property models, namely the Tandon \& Weng model [33], and the orientation averaging procedures. Although this model will introduce error to results, similar to the interaction coefficient it will be small, especially considering the success several researchers have had in providing most accurate results, when employed for injection-moulded short fibre reinforced composites.

The main source of error involved in the prediction of fibre orientation distributions stems from the fact that 2-D Hele-Shaw flow, based in the lubrication region only, is not adequate to represent the complex flow involved in filling fibre reinforced material into a mould cavity. This type of simulation is suitable, however, for nonreinforced resins, given none of the complex orientation structures exist. Thus, simulations based on generalised Hele-Shaw formulations, will still find applications in today's industry, given the large quantity of base resin products being produced. It will also serve a purpose for thin cavities, as the accuracy improves dramatically for such cavities. Nevertheless, for accurate predictions of fibre orientation and ultimately mechanical properties, fully three-dimensional packages should be employed. With three-dimensional simulations, all three flow regimes, gate, lubrication and fountain flow can be included to provide extremely accurate results. By including this with the advent of more powerful, faster workstations the main limitation of full three-dimensional solutions will be erased. 


\section{Conclusions}

As the flow at the gate region is three-dimensional, it cannot be represented by Hele-Shaw type flow and is normally neglected. However, it has been shown that this region has only a minute effect on the fibre orientation (mostly $\mathrm{a}_{11}$ ) and this effect diminishes away from the gate. Nevertheless, in all cases, a large difference can be observed between predicted and measured results at this particular section. Correspondingly, the fountain flow region cannot be simulated by Hele-Shaw flow either, as the gap-wise velocity is comparable to the in-plane velocity. As a result, the fibre orientation determined through numerical methods predicted a greater degree of alignment in the flow direction than was actually measured. It is also an indication that the fibre-fibre interaction model is not sufficient for this particular geometry and flow condition. Consequently, mechanical property calculations were over-predicted by amounts varying from $52 \%$ to $59 \%$. These calculations are also dependent on assumptions and approximations used in mechanical property models, and orientation averaging procedures. This work has shown that for the simplest of planar geometries, commercial software can predict fibre orientations with reasonable accuracy although the consequences of even small errors in predicting this important variable can mean that mechanical properties are in error by significantly large quantities. This matter is likely to be compounded in more complex three-dimensional injection moulded components.

\section{Acknowledgements}

Financial support for this project has been provided by Enterprise Ireland (Grant Number: PRP00/MI/11). Numerical experiments were carried out on Moldflow Software (Moldflow 3.0, 2002) [34].

\section{References}

[1] Bedworth, DD, Henderson, MR \& Wolfe, PM, 1991, Computer-integrated design and manufacturing, McGraw-Hill, America.

[2] Svensson, N, Shishoo, R \& Gilchrist, MD, 1998, 'Manufacturing of thermoplastic composites from commingled yarns: A review', Journal of Thermoplastic Composite Materials, vol. 11, no. 1, pp. 22-56.

[3] Gilchrist, MD, Kinloch, AJ, Matthews, FL \& Osiyemi, SO, 1996, 'Mechanical performance of carbon-fibreand glass-fibre-reinforced epoxy I-beams. I - Mechanical behaviour', Composites Science and Technology, vol. 56, no. 1 , pp. $37-53$. 
[4] Gilchrist, MD, Kinloch, AJ \& Matthews, FL, 1996, 'Mechanical performance of carbon-fibre- and glass-fibrereinforced epoxy I-beams. II - Fractographic failure observations', Composites Science and Technology, vol. 56, no. 9, pp. 1031-1045.

[5] Bay, RS, 1991, 'Fiber orientation in injected molded composites, a comparison of theory and experiment', Ph.D. Thesis, Dept. Mechanical and Industrial Engineering, University of Illinois at Urbana-Champaign.

[6] Bay, RS \& Tucker, CL, 1991, 'Fibre orientation in simple injection moldings: Part 1 - Theory and numerical methods', ASME Plastics \& Plastic Composites: Material Properties, Part Performance and Process Simulation, vol. 29, pp. 445-471.

[7] Bay, RS \& Tucker, CL, 1991, 'Fibre orientation in simple injection moldings: Part 2 - Experimental results', ASME Plastics \& Plastic Composites: Material Properties, Part Performance and Process Simulation, vol. 29, pp. 473-492.

[8] Gilchrist, MD \& Svensson, N, 1995, 'A fractographic analysis of delamination within multidirectional carbon/epoxy laminates', Composites Science and Technology, vol. 55, no. 2, pp. 195-207.

[9] Svensson, N, Shishoo, R \& Gilchrist, MD, 1998, 'Interlaminar fracture of commingled GF/PET composite laminates', Journal of Composites Materials, vol. 32, no. 20, pp. 1808-1835.

[10] Svensson, N \& Gilchrist, MD, 1998, 'Mixed-mode delamination of multidirectional carbon fiber/epoxy laminates', Mechanics of Composite Materials and Structures, vol. 5, no. 3, pp. 291-307.

[11]Ho, KC \& Jeng, MC, 1996, 'Fibre orientation of short glass fibre reinforced polycarbonate composites under various injection moulding conditions', Plastics, Rubber and Composites Processing and Applications, vol. 25, pp. $469-476$

[12] Singh, P \& Kamal, MR, 1989, 'The effect of processing variables on microstructure of injection molded short fibre reinforced polypropylene composites', Polymer Composites, vol. 10, pp. 344-351.

[13] Kenig, S, 1986, 'Fibre orientation development in molding of polymer composites', Polymer Composites, vol. 7, pp. 50-55.

[14] Viana, JC, Kearney, P \& Cunha, AM, 1998, 'Improving impact strength of injection moulded plates through moulding conditions optimisation: A design of experiments approach', Annual Technical Conference of the Society of Plastic Engineers '98, vol. 1, pp. 646-650.

[15] O’Dowd, F \& Gilchrist, MD, 2004, 'Optimizing molding conditions to improve the quality of injection-molded parts: A design of experiments approach', SME Plastics Insights, vol. 3, no. 4, pp. 1-9. 
[16] O’Dowd, F, 2004, 'Mechanical performance of short fibre-reinforced injection moulded composites', M.Eng.Sc. Thesis, Dept. Mechanical Engineering, University College Dublin.

[17] Advani, SG \& Tucker, CL, 1987, 'The use of tensors to describe and predict fiber orientation in short fiber composites', Journal of Rheology, vol. 31, no. 8,pp. 751-784.

[18] Fischer, G \& Eyerer, P, 1988, 'Measuring spatial orientation of short fibre reinforced thermoplastics by image analysis', Polymer Composites, vol. 9, no. 4, pp. 297-304.

[19] Clarke, AR, Davidson, NC \& Archenhold, G, 1997, 'Mesostructural characterisation of aligned fibre composites', Flow-induced alignment in composite materials, Ed. Papathanasiou, TD \& Guell, DC, Woodhead Publishing.

[20] Stobie, RS, 1980, 'Analysis of astronomical images using moments', Journal of British Interplanetary Society, vol. 33 , pp. 323-326.

[21] Clarke, AR, Davidson, NC \& Archenhold, G, 1993, 'Measurements of fibre direction in reinforced polymer composites', Journal of Microscopy, vol. 171, pp. 69-79.

[22] Hine, PJ, Davidson, N, Duckett, RA \& Ward, IM, 1995, 'Measuring the fibre orientation and modelling the elastic properties of injection-moulded long-glass-fibre-reinforced nylon', Composites Science and Technology, vol. 53, pp. $125-131$.

[23] Mlekusch, B, 1999, 'Fibre orientation in short-fibre-reinforced thermoplastics II. Quantitative measurements by image analysis', Composites Science and Technology, vol. 59, pp. 547-560.

[24] Gupta, M \& Wang, KK, 1993, 'Fibre orientation and mechanical properties of short-fibre-reinforced injectionmolded composites: simulated and experimental results, Polymer Composites, vol. 14, no. 5, pp. 367-382.

[25] Papathanasiou, TD, 1997, 'Flow-induced alignment in injection molding of fiber-reinforced polymer composites', Flow-induced alignment in composite materials, Ed. Papathanasiou, TD \& Guell, DC, Woodhead Publishing.

[26] Tucker, CL, 1991, 'Flow regimes for fibre suspensions in narrow gaps', Journal of Non-Newtonian Fluid Mechanics, vol. 39, no. 3, pp. 239-268.

[27]Hieber, CA \& Shen, SF, 1980, 'A finite-element/finite-difference simulation of the injection-molding filling process', Journal of Non-Newtonian Fluid Mechanics, vol. 7, pp. 1-32.

[28] Jeffery, GB, 1923, 'The motion of ellipsoidal particles immersed in a viscous fluid', Proceedings of the Royal Society, Series A, vol. 102, pp. 161-179. 
[29]Folgar, F \& Tucker, CL, 1984, 'Orientation behaviour of fibers in concentrated suspensions', Journal of Reinforced Plastic Composites, vol. 3, pp. 98-119.

[30] Advani, SG \& Tucker, CL, 1990, 'Closure approximations for three-dimensional structure tensors', Journal of Rheology, vol. 34, no. 3, pp. 367-385.

[31]Cintra, JS \& Tucker, CL, 1995, 'Orthotropic closure approximations for flow-induced fiber orientation', Journal of Rheology, vol. 39, no. 6, pp. 1095-1122.

[32] Ni, S 1997, 'Effects of the mesh density on the accuracy of simulation results', Annual Technical Conference of the Society of Plastic Engineers '97, vol. 1, pp. 632-636.

[33] Tandon, GP \& Weng, GJ, 1984, 'The effect of aspect ratio of inclusions on the elastic properties of unidirectionally aligned composites', Polymer Composites, vol. 5, no. 4 pp. 327-333.

[34] Moldflow Plastics Insight, Version 3.0, 2002, Moldflow Pty. Ltd. 


\section{Nomenclature}

$\mathrm{D}_{\mathrm{r}}$

$\mathrm{V}_{\mathrm{f}}$

$\mathrm{D}_{\mathrm{z}}$
Fibre length (m)

Fibre Diameter (m)

Second-order tensor

Fourth-order tensor

Fibre components

Major axis of ellipse

Minor axis of ellipse

Cavity thickness

Fibre concentration

Length of cavity

Interaction coefficient

Rotary diffusivity

Fibre volume fraction

Thickness moment of inertia

Angle of section

Out-of-plane angle

in-plane angle

Kronecker delta function

Probability distribution function

Vorticity tensor

Particle shape dependent parameter

Magnitude of strain rate tensor 
Tables

\begin{tabular}{clcc}
\hline Factor & \multicolumn{1}{c}{ Description } & Level 1 & Level 2 \\
\hline 1 & Injection Time $(\mathrm{s})$ & 4 & 1 \\
\hline 2 & Melt Temperature $\left({ }^{\circ} \mathrm{C}\right)$ & 225 & 270 \\
\hline 3 & Mould Temperature $\left({ }^{\circ} \mathrm{C}\right)$ & 35 & 65 \\
\hline 4 & Injection Pressure Profile $(\%)$ & 50 & 100 \\
\hline 5 & Packing Pressure Profile $(\%)$ & 50 & 100 \\
\hline 6 & Cooling Time (s) & 25 & 55 \\
\hline 7 & Packing Time $(\mathrm{s})$ & 5 & 10 \\
\hline 8 & Cavity Thickness (mm) & 2 & 4 \\
\hline
\end{tabular}

Table 1. Factors and their levels studied in the preliminary experiment.

\begin{tabular}{lccccc}
\hline \multicolumn{1}{c}{ Source } & $\begin{array}{c}\text { Tensile } \\
\text { Strength }\end{array}$ & $\begin{array}{c}\text { Flexure } \\
\text { Strength }\end{array}$ & $\begin{array}{c}\text { Impact } \\
\text { Strength }\end{array}$ & Energy & Weight \\
\hline Main Effects & & & & & \\
1. Injection Time & 0.008 & 1.76 & 0.8 & 0.032 & 7.714 \\
2. Melt Temperature & 0.047 & 0.047 & 34.834 & 0.911 & 6.315 \\
3. Mould Temperature & 0.078 & 0.031 & 85.828 & 0.377 & 3.663 \\
4. Injection Pressure & 0.086 & 0.008 & 8.395 & 0.476 & 0.02 \\
5. Packing Pressure & 7.927 & 7.515 & 3.87 & 0.097 & 33.595 \\
6. Cooling Time & 6.45 & 0.282 & 6.45 & 1.351 & 0.719 \\
7. Packing Time & 3.594 & 3.901 & 3.594 & 0.007 & 10.475 \\
8. Cavity Thickness & 9.242 & 525.942 & 9838.549 & 160.612 & 17885.936 \\
\hline
\end{tabular}

Table 2. Summary of F-Test Statistics from ANOVA of $2_{\text {IV }}^{8-4}$ factorial design. 


\begin{tabular}{clc}
\hline Run & \multicolumn{1}{c}{ Factors } & Values \\
\hline 1 & Melt Temperature & $225^{\circ} \mathrm{C}$ \\
2 & Packing Time & $10 \mathrm{~s}$ \\
3 & Packing Pressure Profile & $50 \%$ \\
4 & Packing Time & $5 \mathrm{~s}$ \\
5 & Packing Pressure Profile & $100 \%$ \\
6 & Cavity Thickness & $5 \mathrm{~mm}$ \\
7 & Melt Temperature & $265^{\circ} \mathrm{C}$ \\
8 & Cavity Thickness & $2 \mathrm{~mm}$ \\
9 & Optimum Conditions & Opt. \\
\hline
\end{tabular}

Table 3. Factors and their levels studied in the Fibre Orientation experiment.

\begin{tabular}{lccc}
\hline Variable & $\begin{array}{c}\text { Predicted Modulus } \\
\text { (MPa) }\end{array}$ & $\begin{array}{c}\text { Measured Modulus } \\
(\mathbf{M P a})\end{array}$ & $\begin{array}{c}\text { Variance } \\
\mathbf{( \% )}\end{array}$ \\
\hline & & & \\
MT - 225 $5^{\circ} \mathrm{C}$ & 7081.5 & 4497 & +57 \\
PT - 5s & 7046.6 & 4539 & +55 \\
PT - 10s & 7063.0 & 4641 & +52 \\
PP - 50\% & 7031.2 & 4497 & +56 \\
PP - 100\% & 7047.6 & 4549 & +55 \\
CT - 5mm & 7064.8 & 4436 & +59 \\
MT - 265 & 7047.6 & 4594 & +53 \\
CT - 2mm & 7075.9 & 4491 & +58 \\
Optimum & 7019.7 & 4577 & +53 \\
\hline
\end{tabular}

Table 4. Comparison between predicted (via Moldflow) and experimentally measured tensile modulus. 


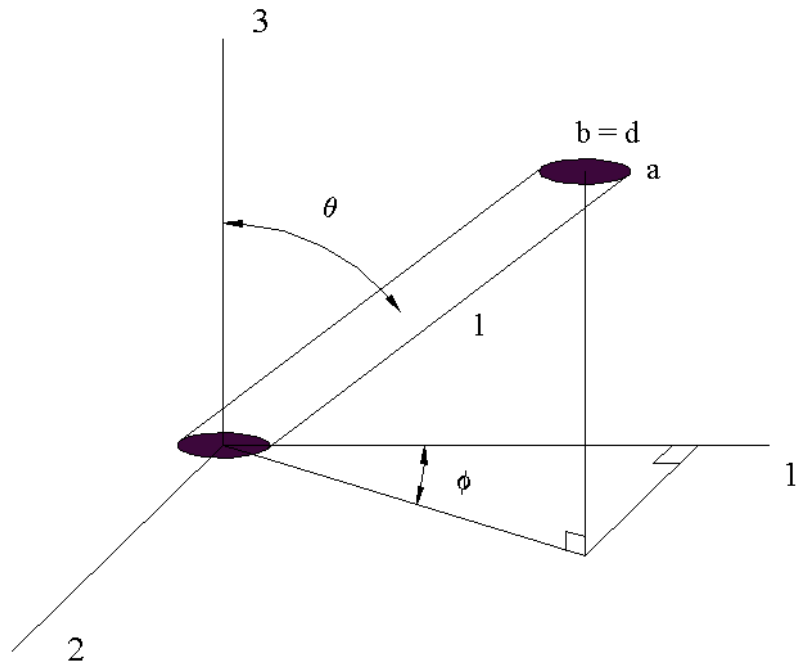

Figure 1. The orientation of a single fibre can be represented in polar coordinates by the out of plane angle, $\theta$, and the in-plane angle, $\phi$. 


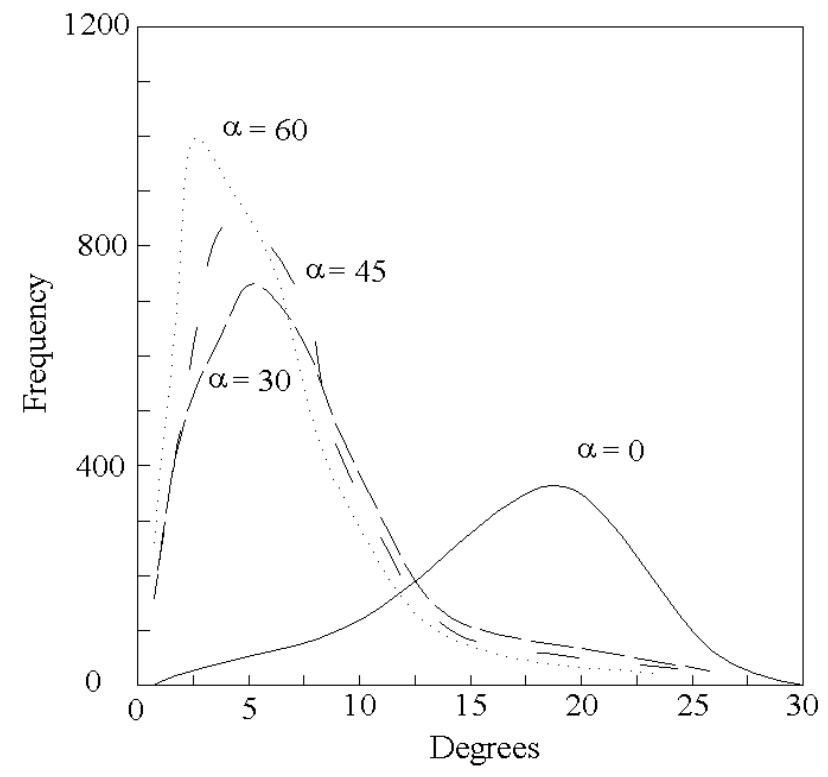

Figure 2. Comparison of the orientation distributions for various sectioning angles, $\alpha$, after Clarke et al. (1997). 


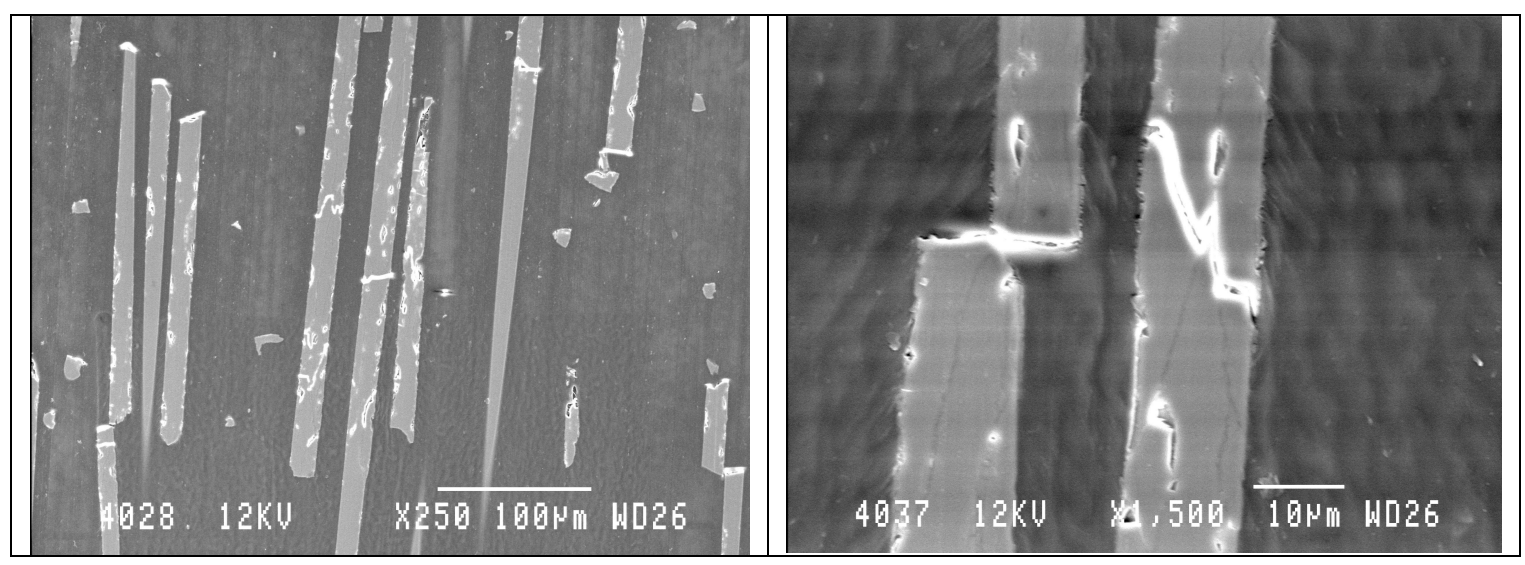

Figures 3 and 4. In-situ SEM micrographs of a sample undergoing deformation. 


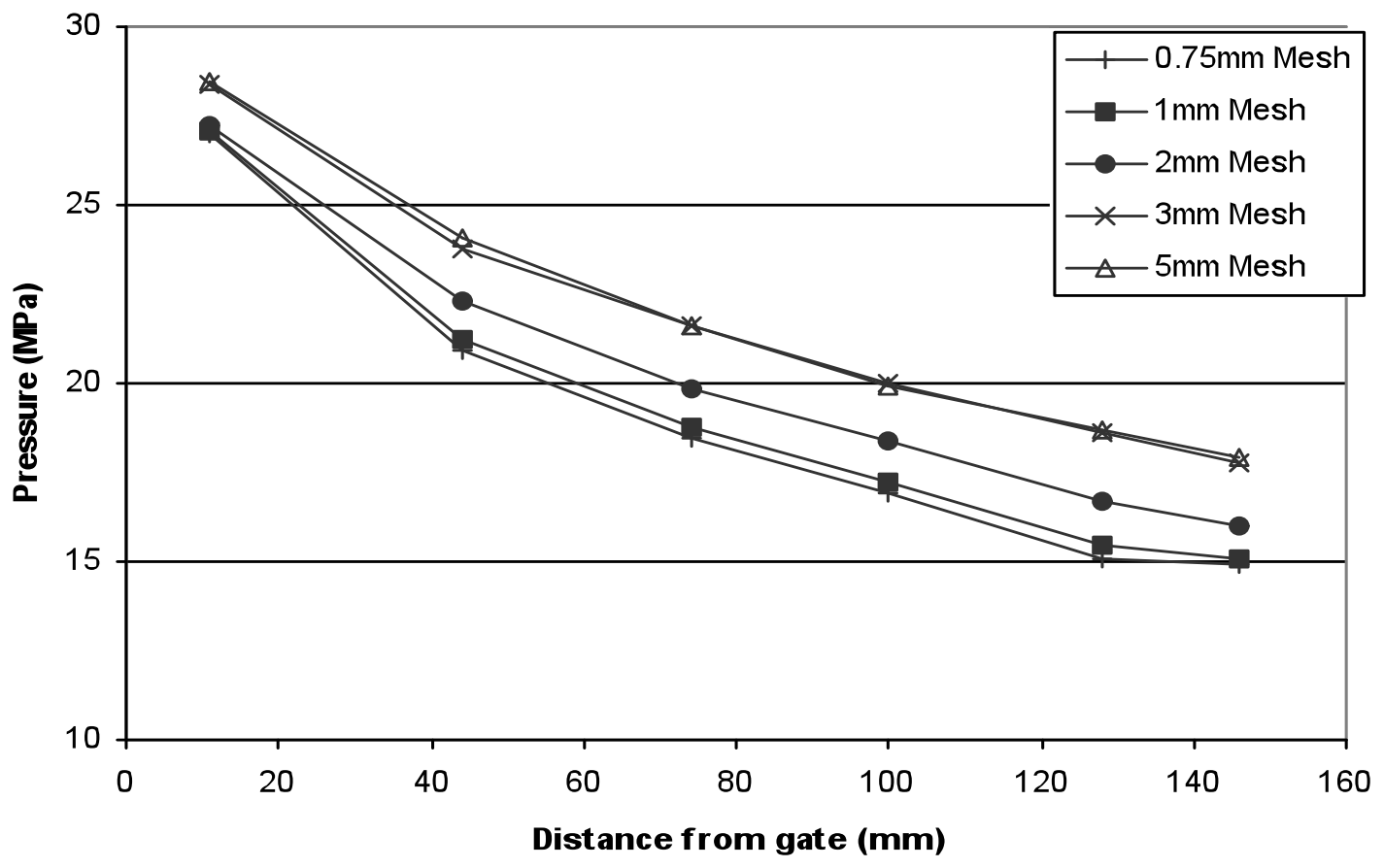

Figure 5. Pressure distribution in cavity at the end of filling. 


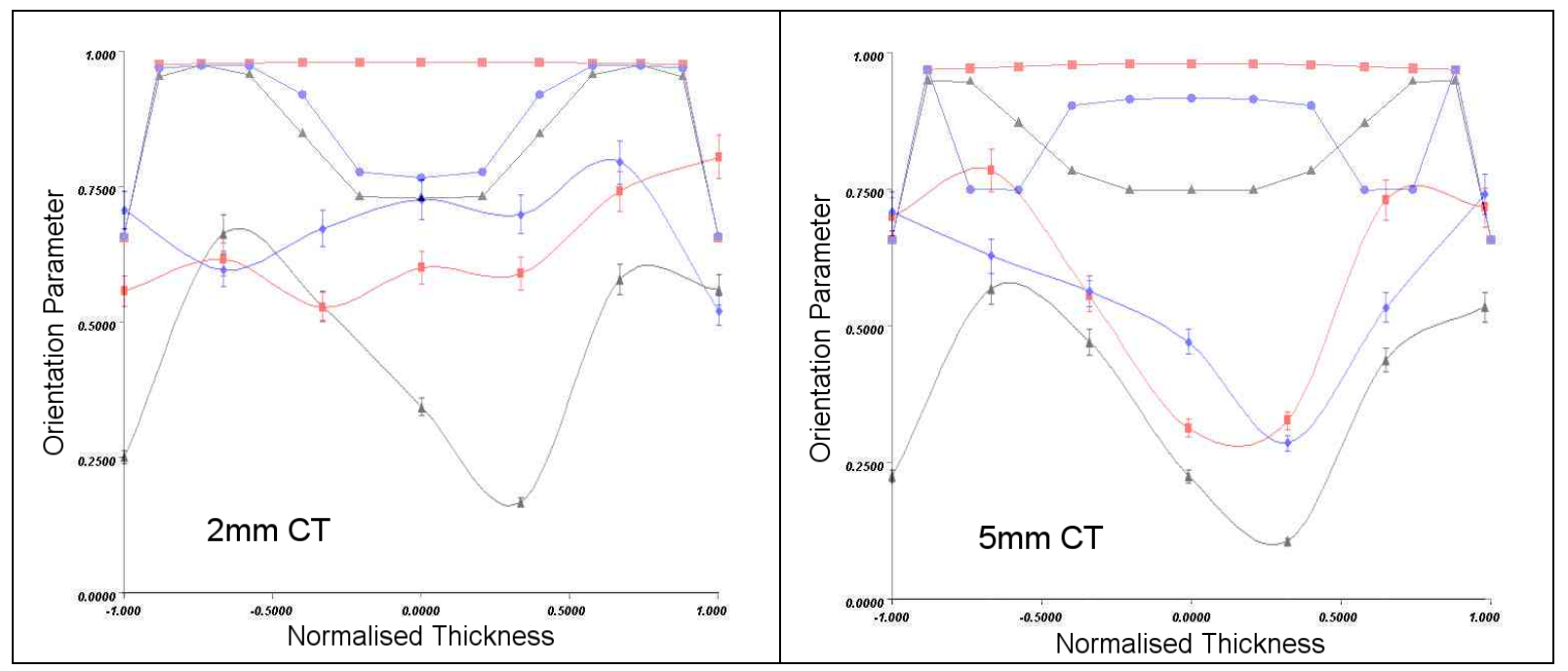

Figures 6 and 7. Predicted orientations (no error bars) vs. measured orientations (including $\pm 5 \%$ error bars) along the length of the cavity, for 2 and $5 \mathrm{~mm}$ cavity thickness (CT). Blue corresponds to a location nearest the gate $(=23$ $\mathrm{mm}$ from the gate), red to the middle of the gauge length (= $75 \mathrm{~mm}$ from the gate), and black to the furthest from the gate $(=135 \mathrm{~mm}$ from the gate) . 


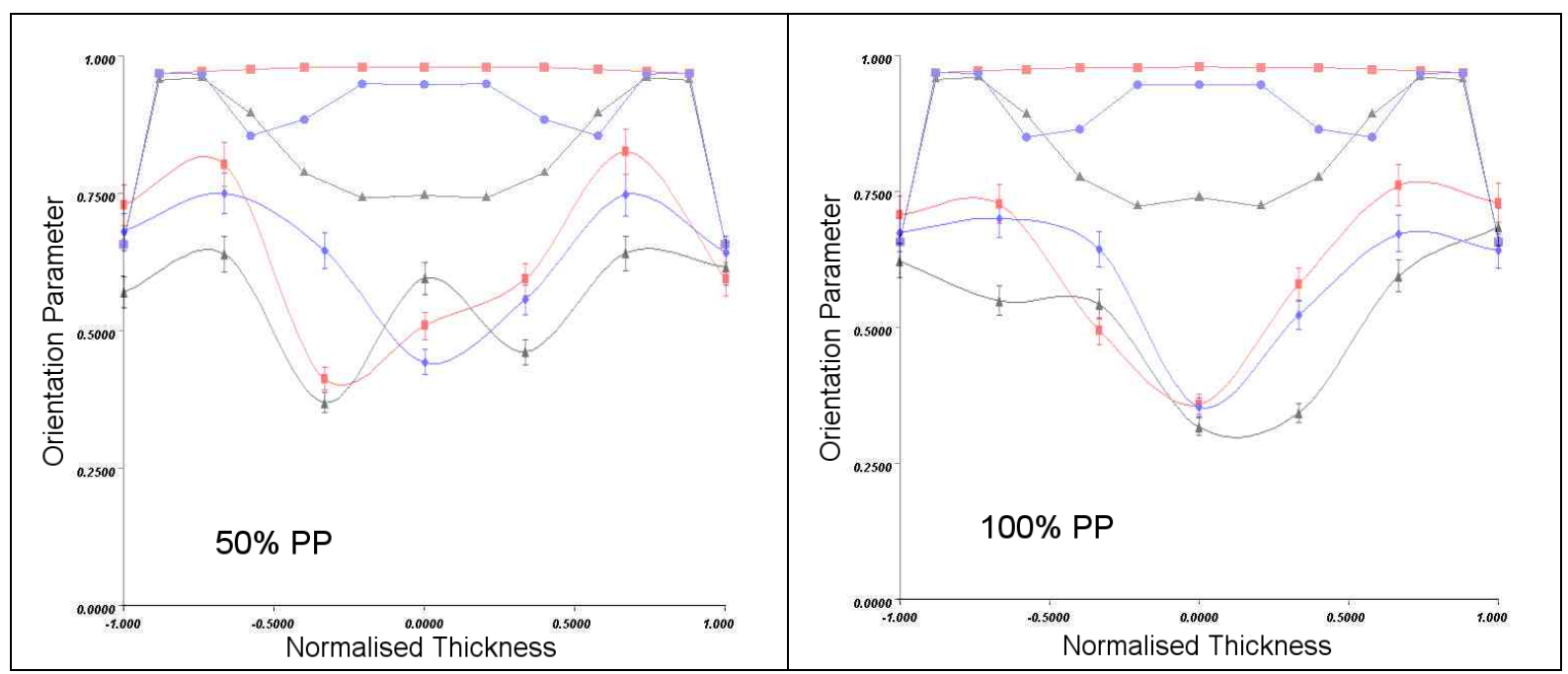

Figures 8 and 9. Predicted orientations (no error bars) vs. measured orientations (including $\pm 5 \%$ error bars) orientations along the length of the cavity, for $50 \%$ and $100 \%$ packing pressure profile (PP). Blue corresponds to a location nearest the gate (=23 $\mathrm{mm}$ from the gate), red to the middle of the gauge length (= $75 \mathrm{~mm}$ from the gate), and black to the furthest from the gate $(=135 \mathrm{~mm}$ from the gate). 


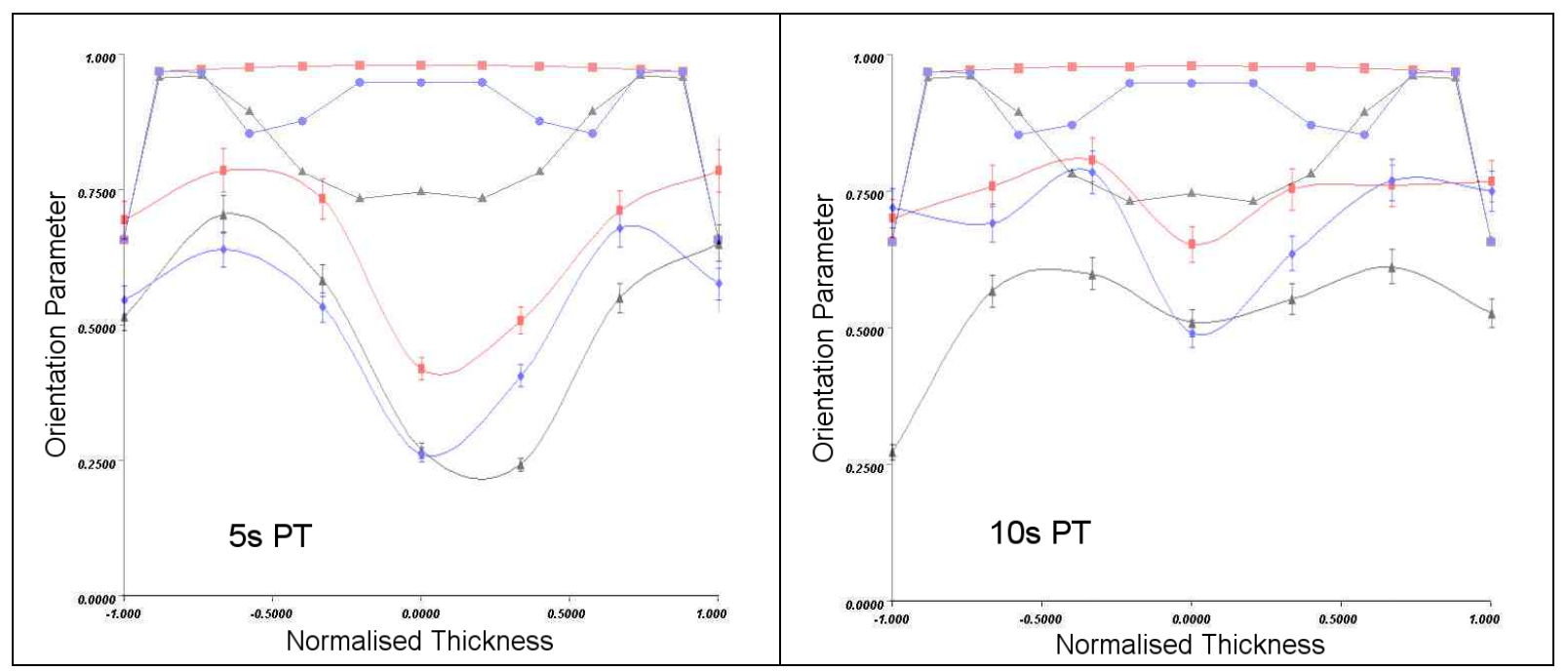

Figures 10 and 11. Predicted orientations (no error bars) vs. measured orientations (including $\pm 5 \%$ error bars) orientations along the length of the cavity, for $5 \mathrm{~s}$ and $10 \mathrm{~s}$ packing time (PT). Blue corresponds to a location nearest the gate (=23 $\mathrm{mm}$ from the gate), red to the middle of the gauge length (=75 $\mathrm{mm}$ from the gate), and black to the furthest from the gate $(=135 \mathrm{~mm}$ from the gate). 


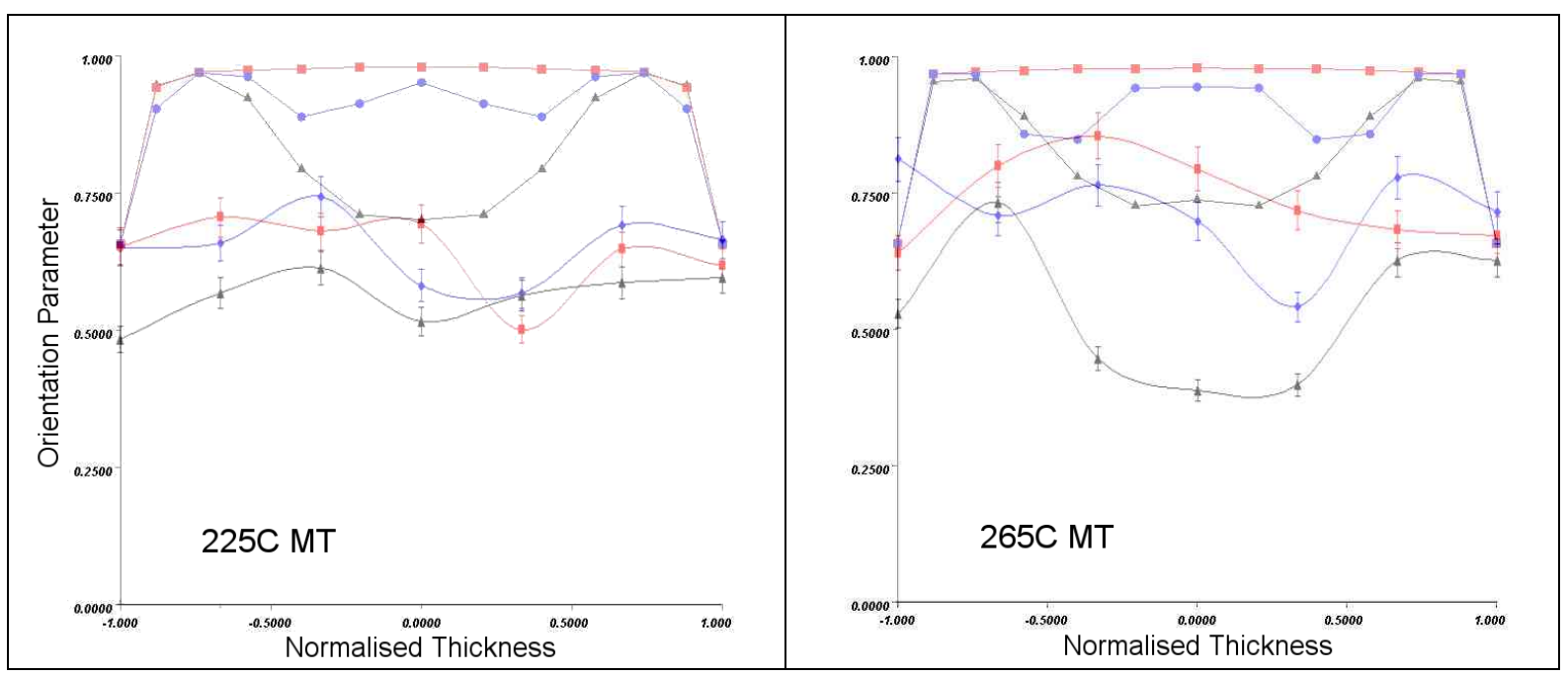

Figures 12 and 13. Predicted orientations (no error bars) vs. measured orientations (including $\pm 5 \%$ error bars) orientations along the length of the cavity, for $225^{\circ} \mathrm{C}$ and $265^{\circ} \mathrm{C}$ melt temperature (MT). Blue corresponds to a location nearest the gate $(=23 \mathrm{~mm}$ from the gate), red to the middle of the gauge length (= $75 \mathrm{~mm}$ from the gate), and black to the furthest from the gate $(=135 \mathrm{~mm}$ from the gate) 


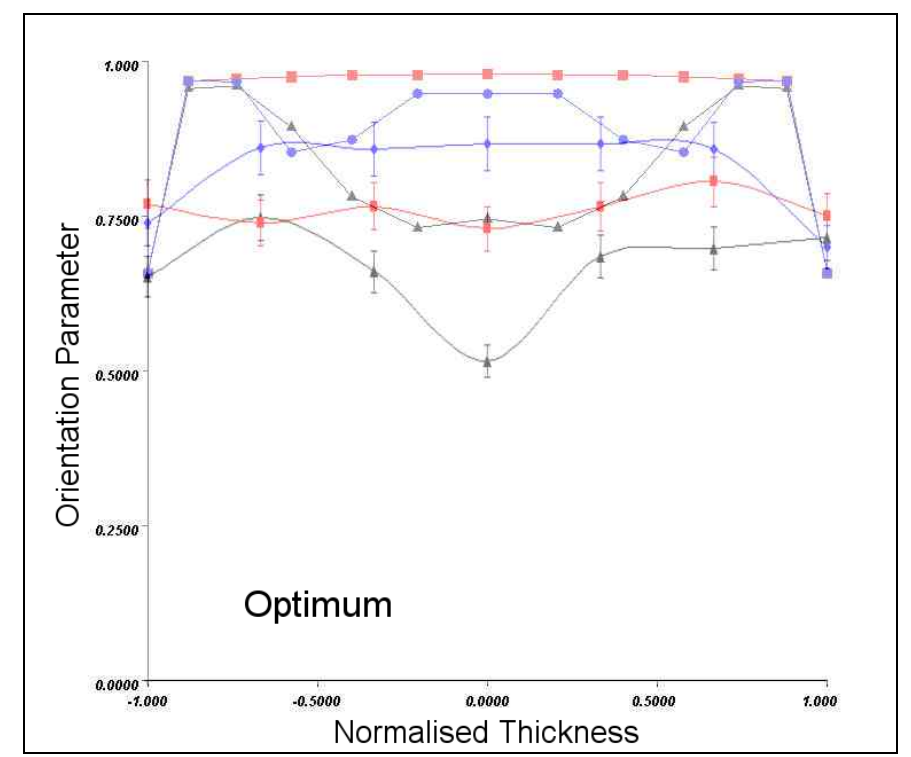

Figures 14. Predicted orientations (no error bars) vs. measured orientations (including $\pm 5 \%$ error bars) orientations along the length of the cavity, for optimum conditions. Blue corresponds to a location nearest the gate $(=23 \mathrm{~mm}$ from the gate), red to the middle of the gauge length (=75 $\mathrm{mm}$ from the gate), and black to the furthest from the gate $(=135 \mathrm{~mm}$ from the gate). 